Research Paper

\title{
Increased Reactive Oxygen Species and Distinct Oxidative Damage in Resveratrol-suppressed Glioblastoma Cells
}

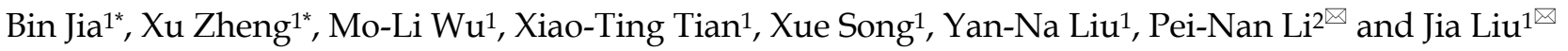 \\ 1. Liaoning Laboratory of Cancer Genetics and Epigenetics and Department of Cell Biology, College of Basic Medical Sciences, Dalian Medical University, Dalian 116044, \\ China. \\ 2. Department of Orthopaedic Surgery, Second Affiliated Hospital, Dalian Medical University, Dalian 116011, China \\ *Equal contributions as co-first authors. \\ $\triangle$ Corresponding authors: Equal contributions of co-corresponding authors. Pei-Nan Li, MD, PhD, Department of Surgery, Second Affiliated Hospital, Dalian Medical \\ University, Dalian 116011, China, delaho@126.com; Jia Liu, MD, PhD, Department of Cell Biology, College of Basic Medical Sciences, Dalian Medical University, Dalian \\ 116044, China, jialiudl@dmu.edu.cn.
}

(C) The author(s). This is an open access article distributed under the terms of the Creative Commons Attribution License (https://creativecommons.org/licenses/by/4.0/). See http://ivyspring.com/terms for full terms and conditions.

Received: 2020.03.01; Accepted: 2020.10.11; Published: 2021.01.01

\begin{abstract}
Background and Aim: Glioblastoma multiforme (GBM) is a highly aggressive brain malignancy that lacks reliable treatments. Resveratrol possesses anti-cancer effects, but its activity against glioblastoma cells is variable for unknown reasons. One mechanism through which anti-cancer drugs exert their effects is oxidative damage caused by increased reactive oxygen species (ROS) production. Thus, the present study examined the relationship between oxidative stress and sensitivity to resveratrol in glioblastoma cells.

Methods: Two GBM cell lines (U251 and LN428) were exposed to $100 \mu \mathrm{M}$ resveratrol for $48 \mathrm{~h}$, and proliferation and apoptosis were assessed. ROS generation was evaluated using 2'-7'-dichlorodihydrofluorescein diacetate-based flow cytometry and fluorescent microscopy. Immunocytochemical staining and western blotting were conducted at regular intervals to profile the expression patterns of superoxide dismutase-2 (SOD2), catalase, caspase-9, caspase-3, and sulfotransferases (SULTs) in untreated and resveratrol-treated GBM cells.

Results: Resveratrol-treated U251 cells, but not resveratrol-treated LN428 cells, exhibited remarkable growth arrest and extensive apoptosis accompanied by elevated intracellular ROS levels and attenuated SOD2 and catalase expression. Mitochondrial impairment and more distinct increases in the expression of activated caspase- 9 and caspase- 3 were detected in U251 cells following resveratrol treatment. The levels of resveratrol metabolic enzymes (SULTIA1 and SULTIC2) were lower in U251 cells than in LN428 cells.

Conclusions: Resveratrol increased ROS generation and induced oxidation-related cellular lesions in U251 cells by activating an ROS-related mitochondrial signal pathway. The levels of SULTs and ROS may indicate the therapeutic outcomes of resveratrol treatment in GBM.
\end{abstract}

Key words: resveratrol; glioblastoma multiforme; reactive oxygen species; oxidative activity; mitochondrial damage

\section{Introduction}

Glioblastoma multiforme (GBM) is the most common brain malignancy in adults [1]. Despite recent advances in aggressive surgery combined with adjuvant therapies [2], the prognosis of advanced GBM remains extremely poor because most patients with GBM die within 15 months after diagnosis and fewer than $3 \%-5 \%$ of patients survive for more than 5 years [3]. Therefore, more effective, less toxic anticancer agents that can penetrate the blood-brain barrier are urgently needed. 
Resveratrol (3,5,4'-trihydroxystilbene) has a wide range of health benefits including chemopreventive, anti-inflammatory, anti-oxidant, and anti-cancer activities [4]. The chemopreventive and anti-cancer effects of this agent have been demonstrated in several cancer types [5]. More importantly, resveratrol can penetrate the blood-brain barrier via simple diffusion, indicating its therapeutic potential in the treatment of brain malignancies [6, 7]. Our previous data revealed that human GBM cells responded differently to resveratrol [8]. For instance, U251 cells were sensitive to $100 \mu \mathrm{M}$ resveratrol in terms of growth arrest and apoptotic cell death, whereas LN428 cells did not respond to the same treatment [8]. Thus, it is important to investigate the underlying factors that explain the differential resveratrol responsiveness of glioblastoma cells for personalized therapy.

Reactive oxygen species (ROS), a group of highly reactive ions and molecules, are generated in the mitochondria as oxygen metabolism by-products [9] and recognized as powerful signaling molecules involved in the cell crisis caused by anti-cancer drugs [10]. In cancer cells, higher ROS levels result in oxidative damage in mitochondria and mitochondrial alterations that trigger the apoptosis cascade by releasing apoptotic signals. Redox regulation plays critical roles in maintaining the survival of malignant cells by reducing ROS levels [11]. Therefore, ROS levels and the efficiency of their dynamic regulation may determine the response of cancer cells to chemotherapy [12-14]. Resveratrol has been reported to have anti-oxidant activity in normal cells, but less data are available for cancer cells [15]. Recently, we found abundant spheroid mitochondria, an indicator of oxidative mitochondrial damage, in resveratroltreated ovarian cancer cells [16]. Our previous studies revealed that the levels of resveratrol-related metabolic enzymes such as sulfotransferases (SULTs) are lower in cancer cells than in their normal counterparts. Moreover, SULT levels are negatively correlated with the sensitivity of glioblastoma cells to resveratrol [17]. These phenomena may explain why resveratrol exerts beneficial effects on normal cells but damaging effects on malignant cells [18]. It is therefore possible that resveratrol increases rather than reduces oxidative stress in cancer cells, presumably because of the poorly operated intracellular resveratrol metabolic machinery in cancer cells. This study examined this speculation using resveratrol-sensitive U251 and resveratrol-resistant LN428 cells.

\section{Materials and Methods}

\section{Chemicals and antibodies}

Resveratrol, dimethylsulfoxide (DMSO) and methylthiazolyldiphenyl-tetrazolium bromide (MTT) were purchased from Sigma-Aldrich Co (St. Louis, MO, USA). TUNEL (terminal dexynucleotidyl transferase (TdT)-mediated dUTP nick end labeling) kit was purchased from Roche Inc., Germany. 2'-7'dichlorodihydrofluorescein-diacetate (DCFH-DA) was purchased from Beyotime Institute of Biotechnology (Jiangsu, China). The rabbit anti-human antibodies against SOD2, Catalase, SULT1A1 and SULT1C2 were purchased from Protein Tech (Chicago, IL, USA), and the rabbit anti-human procaspase-3, active-caspase-3, pro-caspase-9 and activecaspase- 9 antibodies were provided by Abcam (Cambridge, UK).

\section{Cell lines and cell culture}

Human glioblastoma LN428 cell lines were kindly provided by Professor Nicolas de Tribolet, Department of Neurosurgery, Central Hospital University of Laussane, Switzerland and human glioblastoma U251 cell line was provided by the Cell Culture Facility, Chinese Academy of Sciences Cell Bank, Shanghai. The cells were cultured in DMEM (Hyclone Lab, Utah, USA) supplemented with 10\% fetal bovine serum (Gibco Life Science, Grand Island, $\mathrm{NY}, \mathrm{USA}), 100 \mathrm{IU} / \mathrm{ml}$ penicillin, and $100 \mu \mathrm{g} / \mathrm{ml}$ streptomycin under $5 \% \mathrm{CO}_{2}$ and $37^{\circ} \mathrm{C}$ condition. The cells $\left(5 \times 10^{4} / \mathrm{ml}\right)$ were cultured conventional dishes for protein preparation or seeded to the high throughput coverslip preparation dishes (Jet Biofil Biotech Inc., Guangzhou, China) to prepare sufficient cell-bearing coverslips for different experimental purposes. $100 \mu \mathrm{M}$ resveratrol was used to treat the cells.

\section{Cell proliferation and death assays}

Hematoxylin-eosin (H/E) morphological staining was performed on the cell-bearing coverslips with different treatments. The effects of resveratrol on cell proliferation were determined at 24 hour and 48 hour time points by 3-[4,5-Dimethylthiazol-2-yl]-2,5diphenyl-tetrazolium bromide (MTT) assay and shown in OD values. In paralell, TUNEL assay was employed to detect apoptotic cells according to producer's instructions (Promega Corporation, USA).

\section{Ultrastructural examination}

U251 and LN428 cells without and with 48 h 100 $\mu \mathrm{M}$ resveratrol treatment were harvested and fixed in $2.5 \%$ glutaraldehyde $(30 \mathrm{~min}, 50 \mathrm{mM}$ cacodylate buffer, $\mathrm{pH} 7.2$ ) and $2 \% \mathrm{OsO} 4$ (30 mimutes, same 
buffer). Ultra-thin sections $(0.1 \mu \mathrm{M})$ were prepared and examined under a Philips CM100 transmission electron microscope (FEI Company, USA). Images were captured by charge-coupled device camera equipped with TCL-EM-Menu version 3 from Tietz Video and Image Processing Systems (Gaunting, $\mathrm{GmbH}$, Friedrichshafen, Germany) as described elsewhere [18].

\section{Intracellular ROS determination}

2'-7'-dichlorodihydrofluorescein-diacetate

(DCFH-DA) penetrates cells and becomes hydrolyzed to non-fluorescent dichlorodihydrofluorescein (DCFH). DCFH reacts with ROS to form the highly fluorescent dichlorofluorescein (DCF) which can be measured by a flow cytometry. Briefly, U251 and LN428 cells were treated with $100 \mu \mathrm{M}$ resveratrol and collected at $0 \mathrm{~h}, 6 \mathrm{~h}, 12 \mathrm{~h}, 24 \mathrm{~h}$ and $48 \mathrm{~h}$ time points. The cells $\left(2 \times 10^{5}\right)$ were incubated with $10 \mu \mathrm{M}$ DCFHDA (Beyotime Biotech, Jiangsu, China) for 20 minutes at $37^{\circ} \mathrm{C}$ in dark room and washed twice with DMEM. ROS levels were analyzed using a flow cytometer (FACSCalibur, BD Biosciences, San Diego, CA, USA). The cell-bearing coverslips collected at the same time were stained in situ with DCFH-DA and observed and photographed under a fluorescence microscope (Leica, DMI4000B, Germany).

\section{Immunocytochemical staining}

Immunocytochemical staining (ICC) was performed by the method described elsewhere [16]. The rabbit anti-human SOD2, Catalase, rabbit SULT1A1 and SULT1C2 (Proteintech, Chicago, IL, USA) were used in the dilution rates of 1:500, 1:500, $1: 200,1: 150$, respectively. Color reaction was developed using 3, 3'-diaminobenzidinete-trahydrochloride (DAB). According to the labeling intensity, the staining results were evaluated by two independent researchers and scored as negative (-) if no immunolabeling was observed in target cells, weakly positive $(+)$, moderately positive $(++)$, and strongly positive $(+++)$.

\section{Western blot analysis}

Total cellular proteins were prepared from the cells by the method described previously [17]. $30 \mu \mathrm{g}$ sample proteins were separated with $12 \%$ SDS/ PAGE, and transferred to a polyvinylidene difluoride membrance (Amersham, Buckinghamshire, UK). The membrance was blocked with $5 \%$ skimmed milk in $\mathrm{NaCl} /$ Tris-T (10 mM Tris/HCl, pH 8.0, $150 \mathrm{mM} \mathrm{NaCl}$, and $0.5 \%$ Tween-20) at $4{ }^{\circ} \mathrm{C}$ overnight, incubated for 2 hours with the primary antibody and then with horseradish peroxidase-conjugated anti-rat IgG (Zymed Laboratories, San Francisco, CA, USA) for one hour. Immunolabeling was detected with an enhanced chemiluminescence system (Roche Inc., Mannheim, Germany), and visualized with the UVP Bio-spectrum Imaging System (UVP, Upland, CA, USA). $\beta$-actin was used as the internal quantitative control in densitometry analyses.

\section{Statistical analysis}

The experiments were repeated at least for three times and the the normality of the data obtained were analyzed using SPSS software (version 17.0; SPSS, Chicago, IL). The differences in continuous variables were assessed by Student's t-test or one-way ANOVA. Values are presented as the mean \pm standard deviation of triplicate experiments. When required, $P$-values are stated in the figure legends.

\section{Results}

\section{Different resveratrol sensitivities of $\mathrm{U} 251$ and LN428 cells}

The MTT cell proliferation assay revealed that the optical density (OD) of $100 \mu \mathrm{M}$ resveratrol-treated U251 cells was reduced in a time-dependent manner (24 h, $P<0.05 ; 48$ h, $P<0.01)$ in comparison with that of the control cells cultured in medium containing $0.2 \%$ DMSO (Figure 1A). The OD of LN428 cells treated with $100 \mu \mathrm{M}$ resveratrol for $48 \mathrm{~h}$ was reduced by $4.3 \%$ compared with that in control cells $(P>0.05)$.

\section{Extensive apoptosis of resveratrol-treated U251 cells}

A cell viability assay revealed a time-dependent increase of the nonviable fraction of resveratroltreated U251 cells, but not in LN428 cells (Figure 1B). Cytopathological staining using hematoxylin and eosin revealed a distinct apoptotic phenotype in resveratrol-treated U251 cells but not in drug-treated LN428 cells, including cellular shrinkage, chromatin condensation, and the appearance of apoptotic bodies (Figure 1C). Similarly, TUNEL staining demonstrated that the nuclei of resveratrol-treated U251 cells displayed more frequent and stronger green fluorescence labeling than their control counterparts, whereas these findings were not replicated in LN428 cells (Figure 1D).

\section{Mitochondrial alteration in resveratrol-treated U25 1 cells}

Transmission electron microscopy illustrated that in comparison with the intact mitochondria of control cells, double membrane-defined mitochondrial spheroids were commonly observed in resveratrol-treated U251 cells (white arrow) but not in LN428 cells treated under the same experimental condition (Figure 2A). 
A

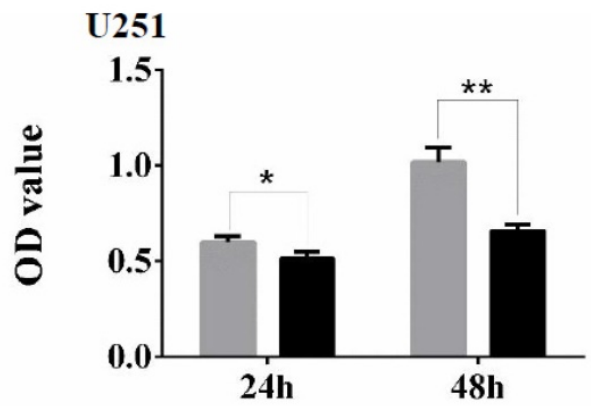

B

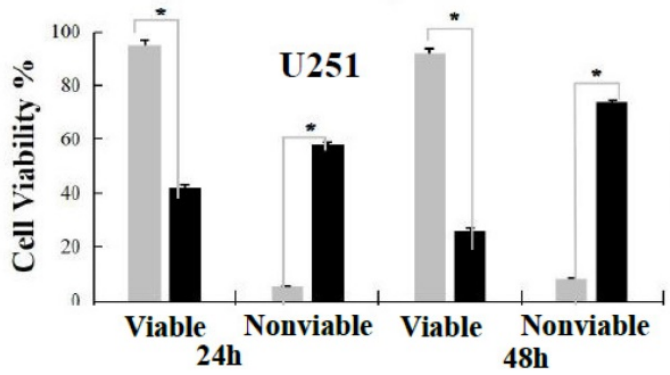

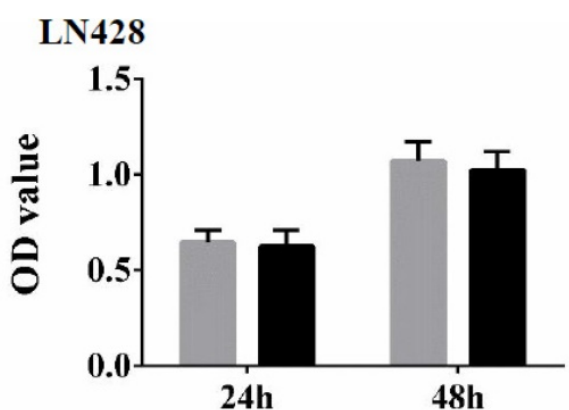

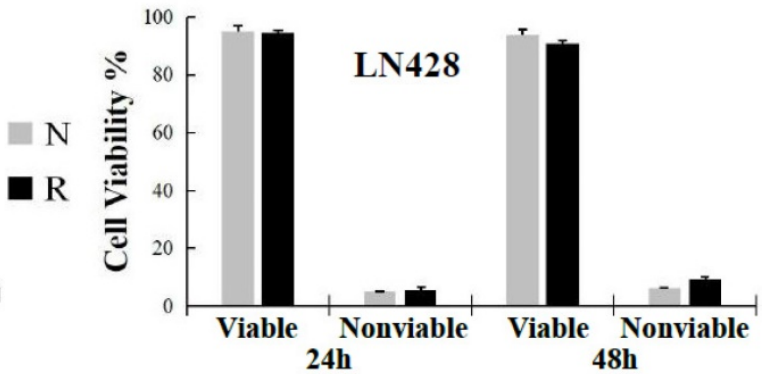

C

oh

$6 h$

$12 \mathrm{~h}$

24h

36h

$48 h$

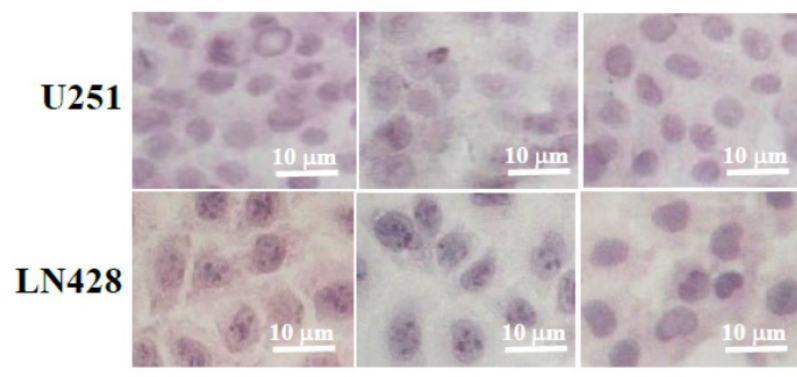

D
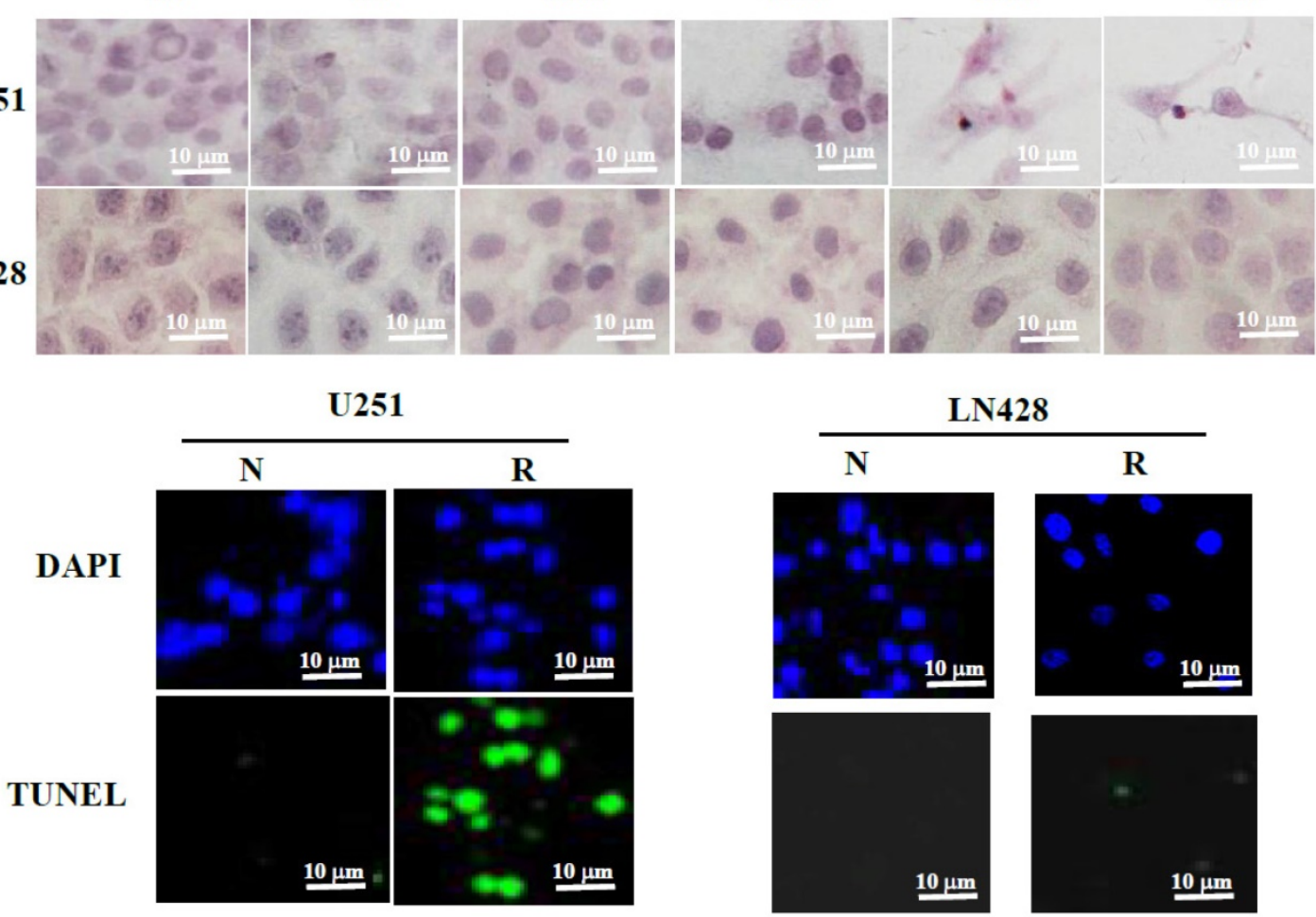

Figure 1. Evaluation of resveratrol sensitivities of U251 and LN428 cells. Resveratrol sensitivities of U251 and LN428 cells were evaluated by MTT assay (A), hematoxylin and eosin morphological staining (B) and fluorescent TUNEL labeling $(\mathbf{C})$. N, without resveratrol treatment; R, treated by $100 \mu M$ resveratrol. *, $P<0.05$ in comparison with $\mathrm{N}$ group; $* *, P<0.01$ in comparison with $\mathrm{N}$ group.

\section{Resveratrol increased ROS levels in U251 cells but not in LN428 cells}

Flow cytometry revealed that compared with the basal fluorescence intensity level at $0 \mathrm{~h}$, ROS generation as reflected using relative fluorescence units was significantly increased $(P<0.01)$ in resveratrol-treated U251 cells at $6(234.04 \pm 9.021), 12$ (329.40 \pm 11.35$), 24(349.09 \pm 11.96), 36$ (306.17 \pm 10.82$)$, and $48 \mathrm{~h}(285.85 \pm 9.701)$. Conversely, no obvious difference of fluorescence intensity was identified between untreated and resveratrol-treated LN428 cells $(P>0.05$; Figure 2B and 2C). The fluorescence microscopy findings were in accordance with the flow 
cytometry results in terms of time-dependent increases of DCF fluorescence intensity in resveratroltreated U251 cells but not in LN428 cells (Figure 2D).

\section{Superoxide dismutase-2 (SOD2) and catalase (CAT) downregulation in U251 cells}

The influence of resveratrol on SOD2 and CAT expression was elucidated via immunocytochemical (ICC) staining (Figure 3A) and western blotting (Figure 3B). The results revealed time-dependent decreases of SOD2 and CAT levels in resveratroltreated U251 cells, whereas their levels remained relatively stable in the presence of resveratrol in LN428 cells.

\section{Activated caspase-9 and caspase- 3 levels in resveratrol-suppressed $\mathrm{U} 251$ cells}

The relevance of the mitochondria-mediated apoptotic pathway to resveratrol-induced apoptosis was elucidated by examining the levels of pro-caspase- 9 and pro-caspase- 3 as well as their enzymatically cleaved forms (activated caspase- 9 and caspase-3) in U251 and LN428 cells. Western blotting (Figure 4A and 4B) illustrated that overall caspase-9 and caspase- 3 levels were decreased by 37.6 and $42.8 \%$, respectively, in $100 \mu \mathrm{M}$ resveratrol-treated U251 cells. However, whereas pro-caspase-9 and pro-caspase-3 levels were slightly decreased by resveratrol treatment, those of activated caspase- 9 and caspase- 3 were increased by 6.15 - and 7.67 -fold, respectively, compared with those in control cells. Meanwhile, pro-caspase- 3 and activated caspase-3 levels were largely unchanged in resveratrol-treated LN428 cells. Activated caspase-9 was undetectable in normally cultured LN428 cells, whereas this protein comprised $14.7 \%$ of the total caspase- 9 pool in resveratrol-treated cells.
A
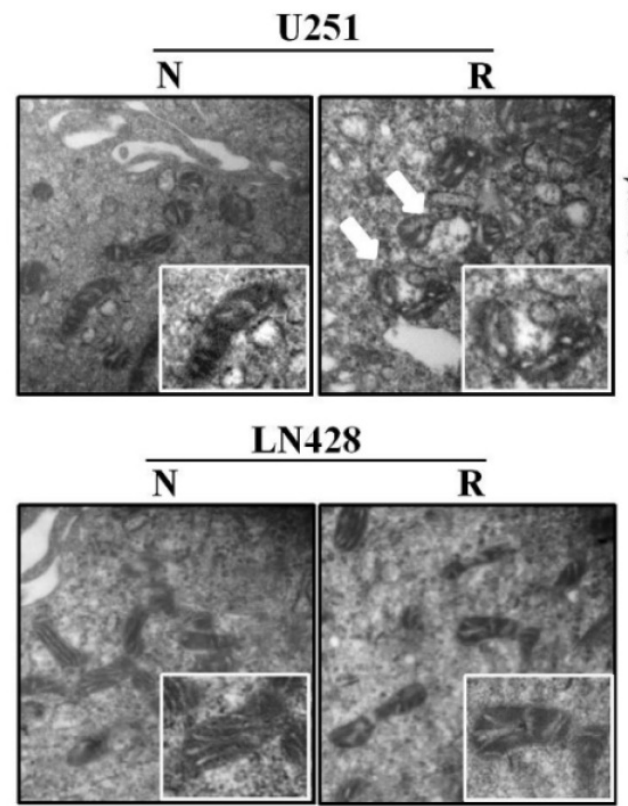

D

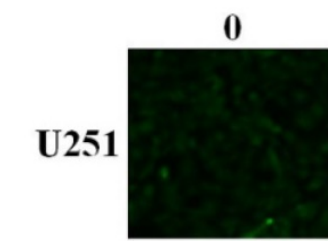

LN428
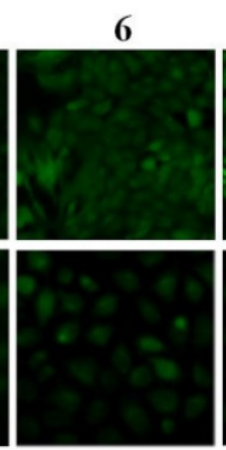

B
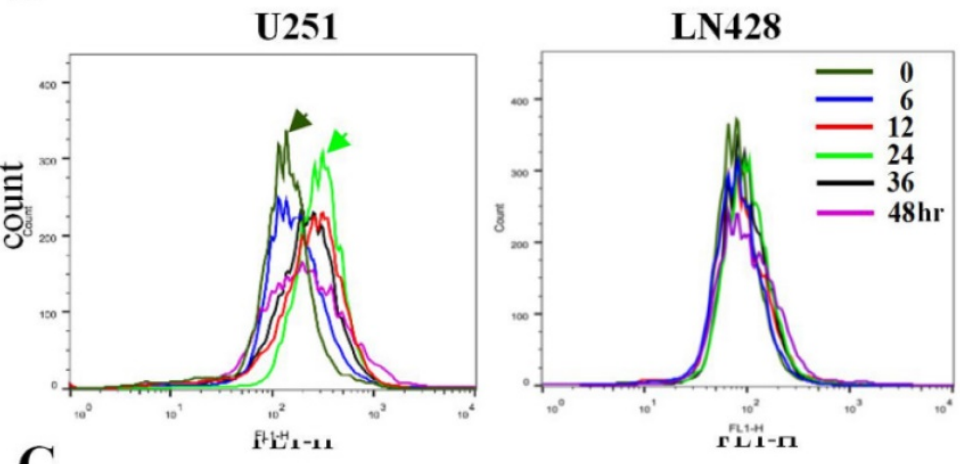

C

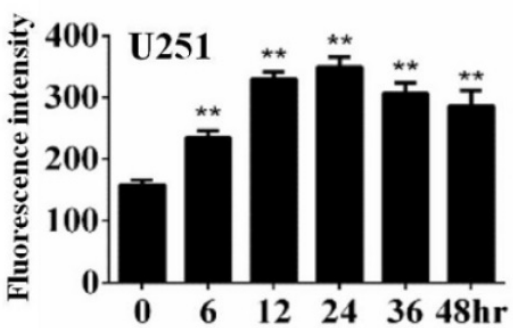

12
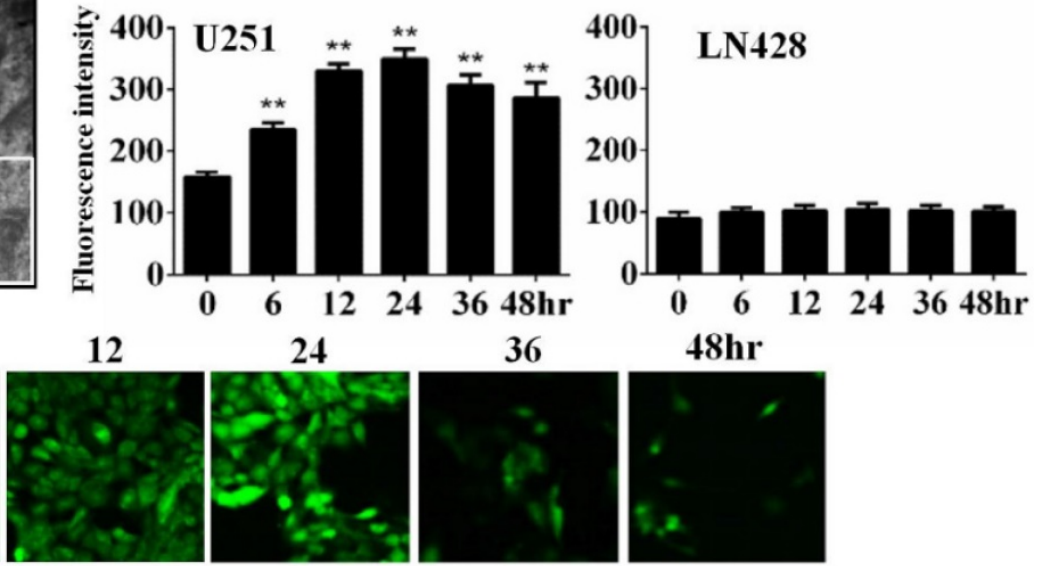

$48 \mathrm{hr}$

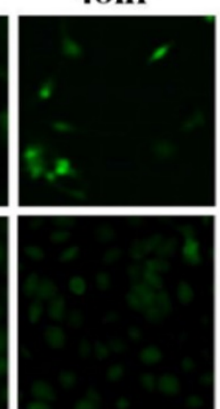

Figure 2. Mitochondrial spheroid formation and reactive oxygen species (ROS) accumulation in resveratrol-sensitive U25 I cells. (A) Transmission electron microscopic examination $(\times 40,000)$ of the double membrane-defined mitochondrial spheroids (white arrow) in resveratrol-treated $\mathrm{U} 251$ cells. $\mathrm{N}$, without resveratrol treatment; $\mathrm{R}$, treated with $100 \mu \mathrm{M}$ resveratrol for $48 \mathrm{~h}$. (B) The cells were treated with $100 \mu \mathrm{M}$ resveratrol for $0,6,12,24,36$, or $48 \mathrm{~h}$ and stained with 2'-7'-dichlorodihydrofluorescein diacetate. Variation of the intracellular fluorescence intensity in U251 and LN428 cells was determined using flow cytometry. (C) Fluorescence intensity analysis of U251 and LN428 cells. (D) ROS levels in U251 and LN428 cells were measured using fluorescence microscopy. All data are presented as the mean \pm SD of three independent experiments. Compared with the 0 h sample, $*, P<0.05 ; * *, P<0.01$. 
A

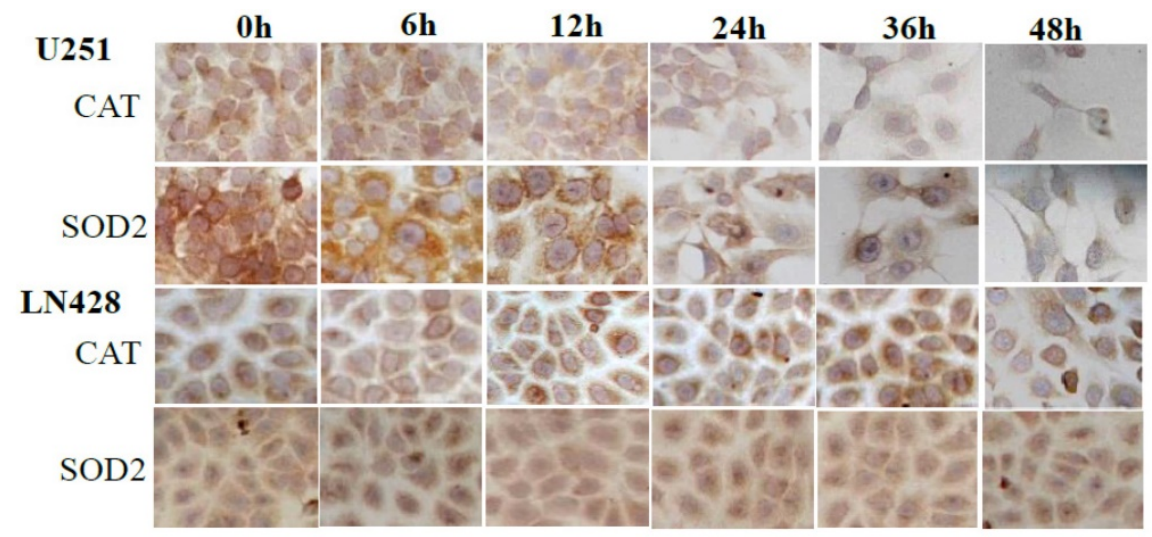

B
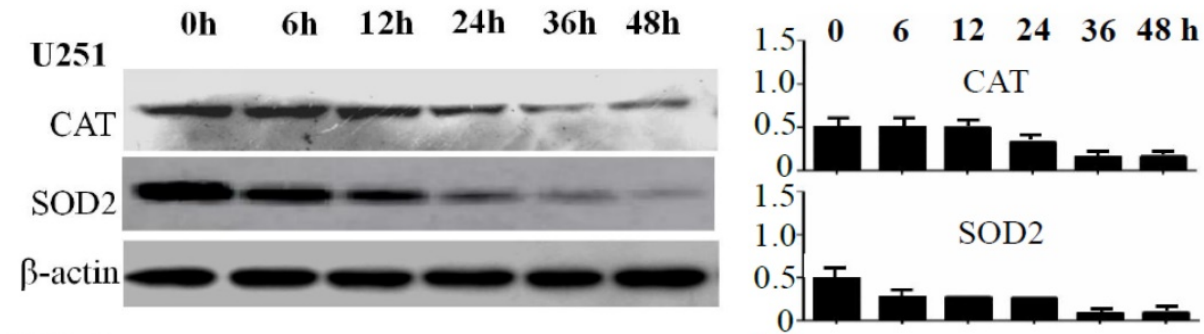

LN428
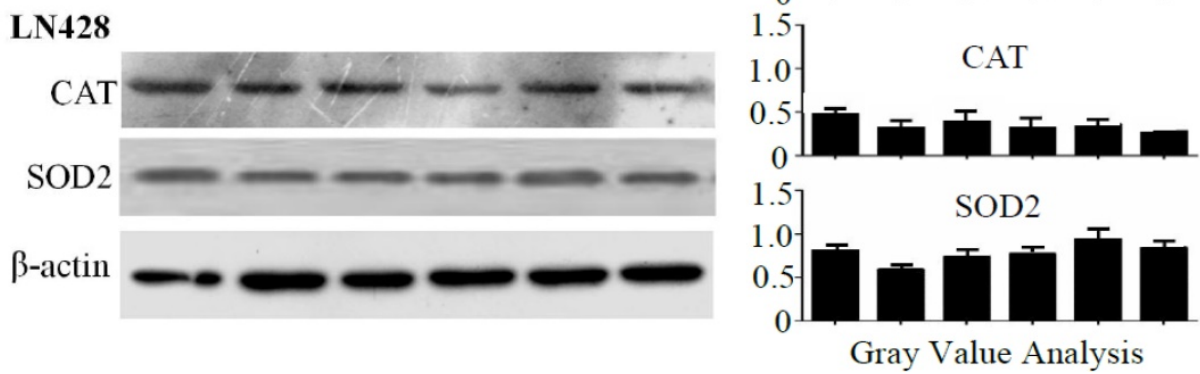

Figure 3. Sequential analyses of superoxide dismutase-2 (SOD2) and catalase (CAT) levels in resveratrol-treated U251 and LN428 cells. (A) Immunocytochemical evaluation of SOD2 and CAT expression in U251 and LN428 cells treated with $100 \mu \mathrm{M}$ resveratrol for 0, 6, 12, 24, 36, or 48 h. (B) Western blot and gray density analyses of SOD2 and CAT expression in U251 and LN428 cells. $\beta$-actin was used as the quantitative control.

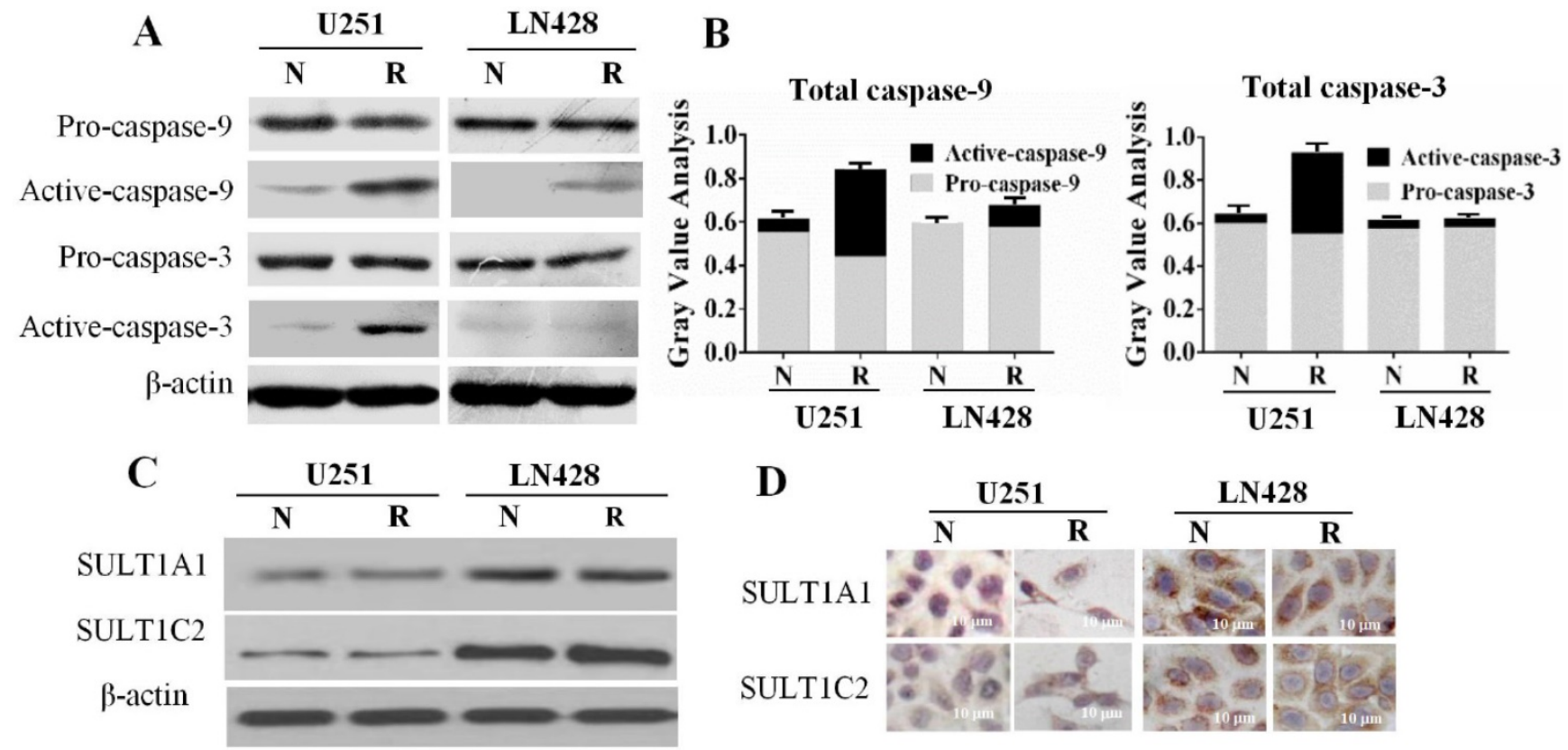

Figure 4. Caspase- 9 and caspase- 3 levels and sulfotransferase $1 \mathrm{Al}$ (SULTIAI) and SULTIC2 expression patterns in untreated and resveratrol-treated U251 and LN428 cells. (A) Western blot evaluation of pro-caspase-9, pro-caspase-3, activated caspase-9, and activated caspase-3 U251 and LN428 cells cultured normally (N) and in the presence of $100 \mu \mathrm{M}$ resveratrol for $48 \mathrm{~h}(\mathrm{R})$. (B) Fractionation of pro-caspase-9, pro-caspase-3, activated caspase-9, and activated caspase-3 in normally cultured (N) and resveratrol-treated (R) U251 and LN428 cells according to the western blot results. (C-D) Western blot and immunocytochemical demonstration of SULT1A1 and SULT1C2 downregulation in U251 cells compared with that in LN428 cells before (N) and after treatment with $100 \mu M$ resveratrol for $48 \mathrm{~h}(\mathrm{R})$. 


\section{Differential SULTIA1 and SULT1C2 expression in GBM cells}

SULTs, the major metabolic enzymes for transresveratrol in human cells, have a negative correlation with chemosensitivity [18]. Therefore, the expression of the SULT1A1 and SULT1C2 isoenzymes in the two GBM cell lines in the presence and absence of resveratrol was examined. The results of western blotting (Figure 4C) demonstrated that SULT1A1 and SULT1C2 were expressed in normally cultured U251 and LN428 cells, and their levels remained almost unchanged after resveratrol treatment. ICC staining revealed that SULT1A1 and SULT1C2 staining was weakly positive $(+)$ in both normally cultured and resveratrol-treated U251 cells. Conversely, SULT1A1 was strongly positive $(+++)$ and SULT1C2 was moderately positive $(++)$ in LN428 cells irrespective of resveratrol treatment (Figure $4 \mathrm{D}$ ).

\section{Discussion}

Intracellular ROS-induced oxidative damage is one of the biological effects of anti-cancer drugs [19-21], as reflected by mitochondrial swelling [22] and spheroid mitochondrion formation [23]. Resveratrol has multifaceted biological activities including anti-oxidant and anti-cancer effects [24]. Our previous studies revealed that resveratrol has little influence on the growth of normal cells [25], suggesting that the molecular elements related with the effects of resveratrol may be altered in cancer cells, resulting in different biological consequences including oxidative stress. To elucidate this speculation, it is necessary to select resveratrolsensitive and resveratrol-insensitive cancer cells and measure ROS levels and oxidation-related events after resveratrol treatment.

To date, no efficient therapeutic regimen has been developed for patients with GBM. The nontoxic properties of resveratrol and its ability to cross the blood-brain barrier [26] support the potential usefulness of this polyphenol compound in the management of brain malignancies. However, only certain GBM cell lines, such as U251 cells, are sensitive to resveratrol. Because oxidation-induced mitochondrial spheriods were commonly found in resveratrol-suppressed ovarian cancers [16], we speculated that such biological events may exist in resveratrol-treated GBM cells and that they are related to the sensitivity to this drug. Therefore, the oxidative statuses of resveratrol-sensitive U251 cells and resveratrol-resistant LN428 cells were elucidated by sequentially measuring ROS levels during $48 \mathrm{~h}$ of resveratrol treatment. The results demonstrated that ROS levels were elevated in resveratrol-treated U251 cells in a time-dependent manner, whereas ROS levels remained low and stable in resveratrol-treated LN428 cells. These findings revealed the ability of resveratrol to increase oxidative stress in sensitive GBM cells and revealed the positive correlation of ROS levels with resveratrol sensitivity. The distinct spheroid mitochondrion formation found in resveratrol-treated U251 cells but not in drug-treated LN428 cells further supported this notion because this structural alteration is regarded as the consequence of ROS-induced oxidative damage and it could induce mitochondrial dysfunction $[27,28]$.

The cellular redox balance is typically maintained by a powerful anti-oxidant system in which SOD2 and CAT play active roles [29, 30]. Because of the increased ROS levels in resveratrolsensitive U251 cells but not in resveratrol-resistant LN428 cells, SOD2 and CAT levels in the two cell lines were analyzed before and after resveratrol treatment. SOD2 and CAT levels were similar in the cell lines under normal culture conditions. After drug treatment, SOD2 and CAT expression was downregulated in U251 cells in a time-dependent manner. This phenomenon thus indicates the inefficiency of the anti-oxidant defense system in resveratrol-treated U251 cells, which may explain the resulting ROS accumulation and oxidative damage [31]. Antioxidation is one of the beneficial effects of resveratrol on normal cells [32], but this effect is less commonly described in cancer cells [15]. Our results clearly demonstrated that resveratrol significantly increases ROS levels and downregulates SOD2 and CAT production. Because these events increased oxidative stress and only occurred in resveratrol-sensitive GBM cells, it is reasonable to consider that the redox state of GBM cells is one of the elements explaining their resveratrol sensitivity. It would be worthwhile to investigate other oxidative stress factors such as GPX1 and SOD1 to further support this notion.

ROS accumulation is an early step in mitochondria-mediated apoptosis [30, 32]. ROS overproduction causes mitochondrial collapse, leading to the release of cytochrome $\mathrm{c}$ and activation of caspase cascades through the conversion of procaspase- 9 and pro-caspase- 3 to their activated forms. Meanwhile, the mitochondria tend to fuse together to form spheroids as a protective response to the increased oxidative stress $[16,31]$. Therefore, the mitochondrial structure and caspase- 3 and caspase- 9 expression in the presence and absence of resveratrol were investigated in GBM cells. The results revealed distinct spheroid mitochondrion formation in resveratrol-sensitive U251 cells, and this event appeared within $6 \mathrm{~h}$ of resveratrol exposure and before the onset of apoptosis. In accordance, the 
fractions of activated caspase- 9 and caspase- 3 were remarkably increased in resveratrol-treated U251 cells. It has been recognized that the release of cytochrome $\mathrm{c}$ and activation of the caspase-mediated apoptosis cascade are linked to ROS-induced mitochondrial damage [33]. These findings thus provide further evidence of resveratrol-enhanced oxidative stress and its correlation with growth suppression and apoptosis in GBM cells. We also noticed that despite being undetectable in control cells, activated caspase- 9 comprised $14.7 \%$ of the total caspase-9 pool in resveratrol-treated LN428 cells, a similar level as that $(10.3 \%)$ found in normally cultured U251 cells. Because the fraction of activated caspase- 9 was increased by 6.15 -fold accompanied by the appearance of mitochondrial spheroids in resveratrol-treated U251 cells, it is possible that the biological effects of resveratrol on LN428 cells are limited, and induction of activated caspase-9 in these cells may not be sufficient to promote apoptosis.

Resveratrol suppresses cancer cell growth without affecting normal cells because the availability of resveratrol in normal cells is well controlled by efficient biotransformation machinery operated by metabolic enzymes such as SULTs [8, 17, 24]. Alternatively, SULT levels are negatively correlated with the anti-cancer efficacy of resveratrol [34], including oxidative stress as described in the current study. For this reason, SULT1A2 and SULT1C2 expression patterns were assessed in control and resveratrol-treated U251 and LN428 cells. The results revealed that basal SULT1A1 and SULT1C2 expression was lower in U251 cells than in LN428 cells, and this situation was unchanged after resveratrol treatment. These results suggest that the decreased metabolic efficiency in U251 cells may increase the intracellular bioavailability of resveratrol and therefore result in oxidative damage instead of anti-oxidant effects. SOD2 and CAT downregulation in resveratrol-treated U251 GBM cells can aggravate oxidative stress, although the underlying mechanism leading to their reduction remains to be investigated.

Taken together, this study demonstrated distinctive growth arrest and apoptosis in resveratroltreated U251 cells accompanied by remarkably increased ROS levels, caspase- 9 and caspase-3 activation, spheroid mitochondrion formation, and reduced SOD2 and CAT production. By contrast, those events did not occur in resveratrol-treated LN428 cells. These findings thus indicate that oxidative stress is one of the cancer-suppressive effects of resveratrol. In this context, ROS levels represent a new parameter for predicting the efficacy of resveratrol against GBMs.

\section{Acknowledgments}

This work was supported by the grants from National Natural Science Foundation of China (Nos. 81672945 and 81450016), Research Fund for $\mathrm{PhD}$ supervisors from National Education Department of China (20122105110005), and a special grant for creative research from South China University of Technology. We thank Joe Barber Jr., PhD (www.liwenbianji.cn/ac) for editing the English text of a draft of this manuscript.

\section{Competing Interests}

The authors have declared that no competing interest exists.

\section{References}

1. Ohgaki H, Kleihues P. Epidemiology and etiology of gliomas. Acta Neuropathol. 2005; 109: 93-108.

2. Palanichamy K, Erkkinen M, Chakravarti A. Predictive and prognostic markers in human glioblastomas. Curr Treat Options Oncol. 2006; 7: 490-504.

3. Xia SL, Wu ML, Li H, et al. CRABP-II- and FABP5-independent responsiveness of human glioblastoma cells to all-trans retinoic acid. Oncotarget. 2015; 6: 5889-5902.

4. Pan Y, Zhang H, Zheng Y, et al. Resveratrol exerts antioxidant effects by activating SIRT2 to deacetylate Prx1. Biochem. 2017; 56: 6325-6328.

5. Ndiaye M, Kumar R, Ahmad N. Resveratrol in cancer management: where are we and where we go from here? Annals NY Acad Sci. 2011; 1215: 144-149.

6. Song $\mathrm{X}, \mathrm{Shu} \mathrm{XH}$, Lin $\mathrm{S}$, et al. Lumbar puncture-administered resveratrol inhibits STAT3 activation, enhancing autophagy and apoptosis in orthotopic rat glioblastomas. Oncotarget. 2016; 7: 75790-75799.

7. Mokni M, Elkahoui S, Limam F, et al. Effect of resveratrol on antioxidant enzyme activities in the brain of healthy rat. Neurochem Res. 2007; 32: 981-987.

8. Sun Z, Shi S, Li H, et al. Evaluation of resveratrol sensitivities and metabolic patterns in human and rat glioblastoma cells. Cancer Chemoth Pharmacol. 2013; 72: 965-973.

9. Quinlan CL, Orr AL, Perevoshchikova IV, et al. Mitochondrial complex II can generate reactive oxygen species at high rates in both the forward and reverse reactions. J Biol Chem. 2012; 287: 27255-27264.

10. Zou $\mathrm{Z}$, Chang $\mathrm{H}, \mathrm{Li} \mathrm{H}$, et al. Induction of reactive oxygen species: an emerging approach for cancer therapy. Apoptosis. 2017; 22: 1321-1335.

11. Kim DK, Beaven MA, Kulinski JM, et al. Regulation of reactive oxygen species and the antioxidant protein DJ-1 in mastocytosis. PLoS One. 2016; 11: e0162831.

12. Trachootham D, Lu W, Ogasawara MA, et al. Redox regulation of cell survival. Antioxid Redox Signal. 2008; 10: 1343-1374.

13. Hematpoor A, Paydar M, Liew SY, et al. Phenylpropanoids isolated from Piper sarmentosum Roxb induce apoptosis in breast cancer cells through reactive oxygen species and mitochondrial-dependent pathways. ChemicoBiol Interact. 2018; 279: 210-218.

14. Wilke J, Kawamura $\mathrm{T}$, Watanabe $\mathrm{N}$, et al. Identification of cytotoxic, glutathione-reactive moieties inducing accumulation of reactive oxygen species via glutathione depletion. Bioorg Med Chem. 2018; 26: 1453-1461.

15. de la Lastra CA, Villegas I. Resveratrol as an antioxidant and pro-oxidant agent: mechanisms and clinical implications. Biochem Soc. Transact. 2007; 35: 1156-1160

16. Zhong LX, Zhang Y, Wu ML, et al. Resveratrol and STAT inhibitor enhance autophagy in ovarian cancer cells. Cell Death Disc. 2016; 2: 15071.

17. Sun $\mathrm{Z}, \mathrm{Li} \mathrm{H}$, Shu $\mathrm{XH}$, et al. Distinct sulfonation activities in resveratrolsensitive and resveratrol-insensitive human glioblastoma cells. FEBS J. 2012; 279: 2381-2392.

18. Shu $\mathrm{XH}, \mathrm{Li} H$, Sun $\mathrm{XX}$, et al. Metabolic patterns and biotransformation activities of resveratrol in human glioblastoma cells: relevance with therapeutic efficacies. PLoS One. 2011; 6: e27484.

19. Wasim L, Chopra M. Synergistic anticancer effect of panobinostat and topoisomerase inhibitors through ROS generation and intrinsic apoptotic pathway induction in cervical cancer cells. Cell Oncol (Dordrecht). 2018; 41: 201-212.

20. Skala E, Kowalczyk T, Toma M, et al. Induction of apoptosis in human glioma cell lines of various grades through the ROS-mediated mitochondrial pathway and caspase activation by Rhaponticum carthamoides transformed root extract. Mol Cell Biochem. 2017; 445: 89-97.

21. Maryam A, Mehmood T, Zhang H, et al. Alantolactone induces apoptosis, promotes STAT3 glutathionylation and enhances chemosensitivity of A549 lung adenocarcinoma cells to doxorubicin via oxidative stress. Sci Rep. 2017; 7: 6242 . 
22. Chapa-Dubocq X, Makarov V, Javadov S. Simple kinetic model of mitochondrial swelling in cardiac cells. J Cell Physiol. 2018; 233: 5310-5321

23. Ding WX, Li M, Biazik JM, et al. Electron microscopic analysis of a spherical mitochondrial structure. J Biol Chem. 2012; 287: 42373-42378.

24. Chedea VS, Vicas SI, Sticozzi C, et al. Resveratrol: from diet to topical usage. Food Funct. 2017; 8: 3879-3892.

25. Shu $\mathrm{XH}$, Li H, Sun $\mathrm{Z}$, et al. Identification of metabolic pattern and bioactive form of resveratrol in human medulloblastoma cells. Biochem Pharmacol. 2010; 79: 1516-1525.

26. Tseng SH, Lin SM, Chen JC, et al. Resveratrol suppresses the angiogenesis and tumor growth of gliomas in rats. Clin Cancer Res. 2004; 10: 2190-2202.

27. Hanschmann EM, Godoy JR, Berndt C, et al. Thioredoxins, glutaredoxins, and peroxiredoxins-molecular mechanisms and health significance: from cofactors to antioxidants to redox signaling. Antioxid Redox Signal. 2013; 19: 1539-1605.

28. Galadari S, Rahman A, Pallichankandy S, et al. Reactive oxygen species and cancer paradox: To promote or to suppress? Free Radical Biol Med. 2007; 104: $144-164$

29. Liu X, Chen Z. The pathophysiological role of mitochondrial oxidative stress in lung diseases. J Transl Med. 2017; 15: 207.

30. Kovacic P, Somanathan R. Multifaceted approach to resveratrol bioactivity: Focus on antioxidant action, cell signaling and safety. Oxid Med Cell Longevity. 2010; 3: 86-100.

31. Liu S, Sun Z, Chu P, et al. EGCG protects against homocysteine-induced human umbilical vein endothelial cells apoptosis by modulating mitochondrial-dependent apoptotic signaling and PI3K/Akt/eNOS signaling pathways. Apoptosis. 2017; 22: 672-680.

32. Ohtsuka T, Buchsbaum D, Oliver P, et al. Synergistic induction of tumor cell apoptosis by death receptor antibody and chemotherapy agent through JNK/p38 and mitochondrial death pathway. Oncogene. 2003; 22: 2034-2044.

33. Unuma K, Aki T, Funakoshi T, et al. Extrusion of mitochondrial contents from lipopolysaccharide-stimulated cells: Involvement of autophagy. Autophagy. 2015; 11: 1520-1536.

34. Wu ML, Li H, Yu LJ, et al. Short-term resveratrol exposure causes in vitro and in vivo growth inhibition and apoptosis of bladder cancer cells. PLoS One. 2014; 9: e89806. 